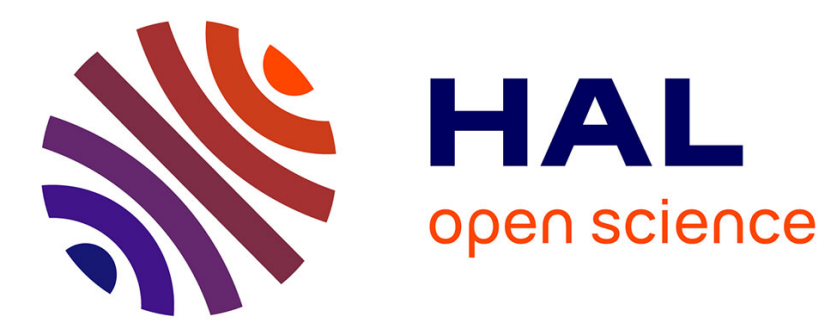

\title{
Particle adhesion to rough surfaces
}

Siddharth Rajupet, Mamadou Sow, Daniel Lacks

\section{To cite this version:}

Siddharth Rajupet, Mamadou Sow, Daniel Lacks. Particle adhesion to rough surfaces. Physical Review E , 2020, 102, pp.012904-1; 012904-9. 10.1103/PhysRevE.102.012904 . hal-02923241

\section{HAL Id: hal-02923241 \\ https://hal.science/hal-02923241}

Submitted on 1 Sep 2020

HAL is a multi-disciplinary open access archive for the deposit and dissemination of scientific research documents, whether they are published or not. The documents may come from teaching and research institutions in France or abroad, or from public or private research centers.
L'archive ouverte pluridisciplinaire HAL, est destinée au dépôt et à la diffusion de documents scientifiques de niveau recherche, publiés ou non, émanant des établissements d'enseignement et de recherche français ou étrangers, des laboratoires publics ou privés. 


\title{
Particle adhesion to rough surfaces
}

\author{
Siddharth Rajupet $\odot,{ }^{1}$ Mamadou Sow, ${ }^{2}$ and Daniel J. Lacks $\oplus^{1}{ }^{1}$ * \\ ${ }^{1}$ Department of Chemical and Biomolecular Engineering, Case Western Reserve University, Cleveland, Ohio 44106, USA \\ ${ }^{2}$ Institut de Radioprotection et de Sûrete Nucleaire (IRSN), Gif-sur-Yvette 91192, France
}

(Received 6 March 2020; revised 20 May 2020; accepted 23 June 2020; published 15 July 2020)

\begin{abstract}
While particle adhesion to smooth surfaces is well understood, real surfaces are not perfectly smooth, and the effects of surface roughness on adhesion are not easily characterized. We develop a theory for the effects of surface roughness on the strength of particle adhesion due to van der Waals forces, in the Derjaguin-MullerToporov (DMT)-type adhesion regime. We first address a well-defined rough surface created by embedding spheres in a smooth substrate, which had been previously examined experimentally. We derive an analytic expression for the adhesive force of particles to this well-defined surface, with the key distinction from the previous work being the inclusion of interactions from surface asperities not in direct contact with the particle. We show that our theory is in good agreement with experimental results in the DMT regime. Within appropriate limits, we extend our theory to general rough surfaces and verify the theory by comparing to the exact numerical results. We show that the interactions from surface asperities not in direct contact with the particle are the dominant contribution to the adhesive force under some conditions, and our theory predicts the experimental and numerical adhesion forces very accurately.
\end{abstract}

DOI: 10.1103/PhysRevE.102.012904

\section{INTRODUCTION}

Systems of fine particles, or "dusts," are characterized by small particle masses and small gravitational forces. Dusts are therefore easily lofted into the air and can travel long distances. On the other hand, dusts easily adhere to surfaces since adhesive forces can dominate over the aerodynamic forces that loft particles. Particle adhesion to surfaces affects a broad spectrum of natural and industrial processes, including granular flow [1], fluidized beds [2], and medical drug delivery $[3,4]$. Of particular interest to us is how particle adhesion affects dust release during a loss of vacuum scenario in tokamak fusion devices; the dust, created by plasma instabilities eroding reactor surfaces [5-7], is radiologically hazardous due to absorption of tritium from the reactor fuel [8-10]. For all of these applications, we must understand the adhesive force between particles and surfaces.

Particle adhesion depends on the nature of particle contact with the surface. In general, a particle deforms elastically to increase the contact area-and thus the attractive interaction - with the surface. The extent of deformation, and how the deformation changes as the particle is lifted off, depends on the balance between the particle-surface attractive forces and the elastic modulus of the particle. When elastic deformation dominates, detachment occurs in a discontinuous "snap-off" manner, as the particle remains deformed with a finite contact area until detachment; this regime is described by Johnson-Kendall-Roberts (JKR) theory [11]. Conversely, when elastic deformation is less important, detachment occurs continuously, as the deformation decreases to zero just before detachment; this regime is described by the Derjaguin-Muller-

*daniel.lacks@case.edu
Toporov (DMT) theory [12]. The transition between these two regimes is described by the Tabor parameter [13],

$$
\mu=\left(\frac{R_{e} \gamma^{2}}{K^{2} d_{c}^{3}}\right)^{1 / 3},
$$

where $\gamma$ is the work of adhesion, $K$ is the effective elastic constant, and $d_{c}$ is the contact separation between the particle and surface (on the order of angstroms). The effective radius is given by $R_{e}=\frac{R R_{2}}{R+R_{2}}$, where $R$ is the radius of the particle and $R_{2}$ is the radius of curvature of the surface that the particle is contacting. The JKR regime occurs at $\mu \gg 1$, and the DMT regime occurs at $\mu \ll 1$. For a sphere interacting with a flat surface, $R_{e}=R$, and the JKR regime occurs for particles that are larger and/or have a lower elastic modulus, while the DMT regime occurs for particles that are smaller and/or have a higher elastic modulus $[14,15]$.

The adhesion between a particle and a smooth surface is well understood, and in the DMT regime, the adhesive force $F_{V}^{S}$ is given by $[16,17]$

$$
F_{V}^{S}=\frac{H R}{6 d_{c}^{2}},
$$

where $H$ is the Hamaker constant. However, real surfaces have at least some roughness. Usually surface roughness reduces the adhesion force between two contacting bodies $[13,18,19]$. In fact, surface roughness has been used as a means to intentionally reduce particle adhesion $[20,21]$. On the other hand, roughness can also enhance particle adhesion $[22,23]$. The reduced adhesion occurs when the particle sits atop surface asperities, while the enhanced adhesion occurs when the particle sits in a valley.

For rough surfaces, the nature of the contact-whether in the JKR or the DMT regime-depends on the amplitude 


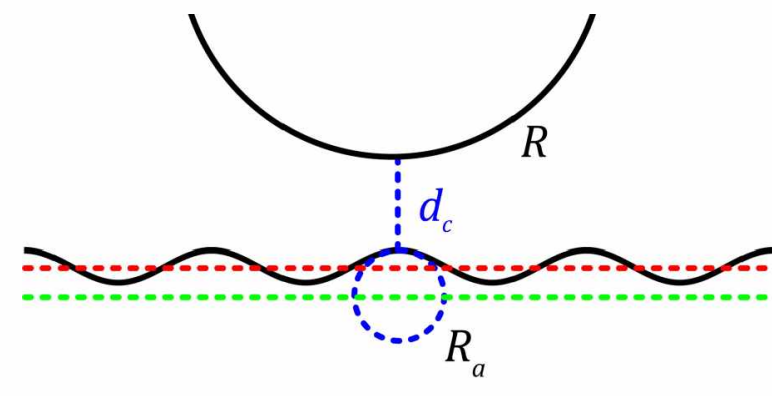

FIG. 1. Using the Rumpf and Rabinovich models, the total van der Waals force between a sphere and a rough surface can be approximated by the sum of two parts: the interaction of the particle with the asperity it is atop, represented as a sphere (blue dashed circle), and the interaction of the particle with a smooth surface at, in the Rumpf model, the center of the spherical asperity (green dashed line), and in the Rabinovich model, the average height of the rough surface (red dashed line).

and wavelength of roughness. In regard to the roughness amplitude, the adhesion changes from JKR-like to DMT-like as the roughness amplitude increases, in the case that the roughness has a short wavelength $(\ll R)$ [24]. In regard to the roughness wavelength, the adhesion undergoes a transition from JKR to DMT behavior as the roughness changes from long to short wavelength [25]. In terms of Eq. (1), the effect of roughness wavelength can be understood in that $R_{e} \approx R$ when the particle interacts with long wavelength roughness, but that $R_{e} \approx R_{2} \ll R$ when the particle interacts with short wavelength roughness.

Simple analytic models have been developed to estimate the van der Waals force between a spherical particle and a rough surface in the DMT regime. These simple analytic models are often advantageous as they are easier to apply and do not require numerical methods to solve; thus, they have been widely used to compare experiments to theory (e.g., [20,22,26-34]). Rumpf considered a particle positioned directly over an asperity, and developed a model for the force that is the sum of two components [35]: (a) the force between the particle and the single asperity located directly below the particle, where the asperity is modeled as a sphere with the same radius of curvature as the asperity, $R_{a}$; and (b) the force between the particle and the remainder of the surface, which is modeled by a smooth surface plane located at distance $R_{a}$ below the peak of the asperity.

This approach, shown schematically in Fig. 1, leads to the force

$$
F_{V, \text { Rumpf }}=F_{V}^{S}\left[\frac{1}{1+R / R_{a}}+\frac{1}{\left(1+R_{a} / d_{c}\right)^{2}}\right],
$$

where the first and second terms represent components (a) and (b) described above, respectively. Rabinovich et al. improved the Rumpf model by noting that the distance parameters in the two terms of Eq. (3) should not be identical - while the distance parameter in the first term is related to the radius of curvature of the asperity $R_{a}$, the distance to the plane representing the rest of the surface is instead related to a roughness amplitude $A[36,37]$. This consideration led to a new expression,

$$
F_{V, \text { Rabinovich }}=F_{V}^{S}\left[\frac{1}{1+R / R_{a}}+\frac{1}{\left(1+A / d_{c}\right)^{2}}\right] .
$$

Furthermore, Rabinovich et al. developed expressions for $R_{a}$ and $A$ in terms of the rms roughness of the surface and the peak-to-peak distance between asperities, $\lambda$ [36], both of which are determinable experimentally from analysis of the surface topography [38]. Several experimental studies have found that the Rabinovich model estimates the adhesion force within an order of magnitude of the experimental data [26-29,34,37].

The Rumpf and Rabinovich models use a planar surface at some effective position to account for portions of the surface beyond the one asperity directly below the particle. The position of this planar surface represents an average due to peaks and valleys in the surface. However, the van der Waals force between atoms weakens very quickly with distance $\left(\sim 1 / r^{7}\right)$. Thus, interactions with peaks of the surface dominate the adhesion force, while interactions with valleys are generally insignificant. This effect is not captured by the interaction of the sphere with a plane at a single intermediate distance. In fact, the Rabinovich model has been shown to significantly underestimate particle adhesion forces when $R$ is large relative to $\lambda$ such that the particle is in close proximity to multiple asperities $[20,27]$. On the other hand, Katainen et al. developed an analytic model that does take into account interaction with multiple asperities. However, the Katainen model is for the JKR regime, and they consider the bottom of the particle to have elastically flattened into a plane of contact area $A_{c}$, and that the particle interacts with all surface asperities that directly contact this plane [27].

There are two distinct approaches to addressing the theory of the interaction between a particle and a rough surface. On the one hand, the interaction can be studied for model surfaces that are well characterized, to enable clear comparison of theory with experiment or numerical solution [20,27,36,39]. On the other hand, analyses can address realistic surface roughness that has more complexity, randomness, and distributions of relevant length scales; such studies represent rough surfaces using a Gaussian distribution of asperity heights [40-43], using a fractal surface such that surface features depend on the magnification of the surface [25,44-48], or by reconstructing the rough surface in Fourier space $[49,50]$. We follow the former approach in this paper.

Here we derive an improved analytic expression to model the adhesive force of a particle to a rough surface in the DMT regime. Our approach incorporates interactions from surface asperities not in direct contact with the particle, which are the dominant contributions in some situations but have been neglected in previous simple models. While previous models were developed based on physical intuition, we begin with well-defined rough surfaces and the fundamental equations for the van der Waals force, and derive an analytic expression for the force. We validate our theory by comparing to experimental results and exact numerical solutions for well-defined rough surfaces. We address the adhesion force, rather than the more general work of adhesion, since the adhesion force 
is more straightforward to compare to previous models and experimental data.

\section{WELL-DEFINED SURFACE: THEORY AND COMPARISON TO EXPERIMENT}

Naturally occurring rough surfaces are inherently irregular and nonuniform, and this complexity prevents clear comparison between experiment and theory. To overcome this challenge, Ramakrishna et al. [20,39] cleverly created a special surface for systematic experiments addressing the effects of surface roughness in a controlled and well-characterized manner, by embedding spheres in a substrate.

Ramakrishna et al. created well-defined rough surfaces by embedding silica spheres with radii of $6 \mathrm{~nm}$ into a silicon substrate such that the embedded spheres protruded by $6 \pm 2$ $\mathrm{nm}$. The surface density of embedded spheres, $\sigma$, varied from $\sigma=0$ to $450 \mu \mathrm{m}^{-2}$. The experiments measured the adhesion force of a 9 - $\mu \mathrm{m}$-radii polyethylene (PE) particle at various $\sigma$, and were performed in $\mathrm{C}_{10} \mathrm{~F}_{18}$ to eliminate capillary forces.

In the experiments of Ramakrishna et al., the particle's large size and low elastic modulus would lead to the adhesion in the JKR regime if the particle was contacting a flat surface. However, on the rough surface, Ramakrishna et al. found that as $\sigma$ decreased from 450 to $245 \mu \mathrm{m}^{-2}$, the contact mechanics transitioned from JKR to DMT [39]. This conclusion was based on the shape of the AFM force-distance curve, which showed continuous lift-off at $\sigma=245 \mu \mathrm{m}^{-2}$ but discontinuous lift-off at $\sigma=450 \mu \mathrm{m}^{-2}$. This finding concurs with previous theories that show that short length-scale roughness causes a transition from the JKR to the DMT regime [25]—at lower $\sigma$ the isolated embedded spheres with nanometer-scale size act as short length-scale roughness, while at high $\sigma$ the nearly constant bed of embedded spheres acts as long lengthscale roughness.

Since our analysis is for the DMT regime, we will use the Ramakrishna et al. results that follow DMT contact mechanics to assess the validity of our results.

\section{A. Theory}

We begin our analysis from fundamental considerations, but point out that the key advance is the derivation of an analytic expression for the adhesive force, in the DMT regime, that incorporates interactions from surface asperities not in direct contact with the particle. While these interactions have been neglected in previous models, we show that they are in some situations the dominant contributions to the adhesive force.

We model the well-defined surface of Ramakrishna et al. as a planar substrate with a grid of spheres of radius $R_{a}$ embedded in the substrate. The substrate is located at $z=0$. The embedded spheres are centered at positions $\left(x_{a, i}, y_{a, i}, z_{a, i}\right)$, where the positions $x_{a, i}, y_{a, i}$ are arranged in a square grid with lattice parameter $\lambda$, and $z_{a, i}=h-R_{a}$ such that the spheres protrude above the substrate by height $h$. The Hamaker constants $H_{\mathrm{Si}}$ and $H_{\mathrm{SiO}_{2}}$ characterize the van der Waals interactions between the polyethylene particle and the silicon substrate and silica embedded spheres, respectively.
The pairwise van der Waals interactions between atoms $j$ and $k$ is described by an attractive energy of the form $-C / r_{j k}^{6}$, where $r_{j k}$ is the distance between atom $j$ and $k$, and $C$ depends on the type of atoms. The total force between the particle and the surface in the $z$ direction (perpendicular to the surface) is obtained by summing all pairwise interatomic interactions,

$$
F_{V}=\sum_{j} \sum_{k} \frac{6 C z_{j k}}{r_{j k}^{8}}
$$

where $j$ loops through atoms in the particle, $k$ loops through atoms in the surface, and $z_{j k}$ is the component of $r_{j k}$ in the $z$ dimension.

In a continuum approximation, the sum in Eq. (5) can be represented as an integral,

$$
F_{V}=\iiint_{X_{p}} \rho_{p} d X_{p} \iiint_{X_{s}} \rho_{s} \frac{6 C\left(z_{p}-z_{s}\right)}{r^{8}} d X_{s},
$$

where $X_{p}$ and $X_{s}$ are the coordinates of the particle and surface, respectively, and $\rho_{p}$ and $\rho_{s}$ are the number densities of the atoms in the particle and surface [17]. For a smooth surface, the integral in Eq. (6) can be solved analytically in the limit $R \gg d_{c}$, with the solution given by Eq. (2).

For the well-defined surface used by Ramakrishna et al., an analytic solution can be determined by splitting the integral into two parts, one part being the force between the particle and the substrate, and the other part being the forces between the particle and each embedded sphere, $i$,

$$
\begin{aligned}
F_{V}= & \iiint_{X_{p}} \rho_{p} d X_{p} \iiint_{X_{\mathrm{sub}}} \rho_{\mathrm{sub}} \frac{6 C_{\mathrm{Si}}\left(z_{p}-z_{\mathrm{sub}}\right)}{r^{8}} d X_{\mathrm{sub}} \\
& +\sum_{i=1}^{\infty} \iiint_{X_{p}} \rho_{p} d X_{p} \iiint_{X_{\mathrm{sp}, i}} \rho_{\mathrm{sp}} \frac{6 C_{\mathrm{SiO}_{2}}\left(z_{p}-z_{\mathrm{sp}, i}\right)}{r_{i}^{8}} \\
& \times d X_{\mathrm{sp}, i},
\end{aligned}
$$

where $X_{\mathrm{sub}}$ represents the coordinates of the substrate and $X_{\mathrm{sp}, i}$ represents the coordinates of the $i$ th spherical asperity. Note that the first term is the interaction between a sphere and a plane, and the second term is the sum of interactions between two spheres.

The analytic solutions to both integrals in Eq. (7) are well known in the limit $d \ll R$ [17], such that Eq. (7) becomes

$$
F_{V}=\frac{H_{\mathrm{Si}} R}{6 d_{s}^{2}}+\frac{H_{\mathrm{SiO}_{2}} R}{6\left(1+R / R_{a}\right)} \sum_{i=1}^{\infty} \frac{z_{i}^{\prime}}{d_{i}^{3}},
$$

where $d_{s}$ is the distance between the particle and the substrate, $d_{i}^{\prime}$ is the separation between the particle and the $i$ th embedded sphere, and $z_{i}^{\prime}$ is the component of $d_{i}^{\prime}$ in the $z$ dimension.

Our strategy for evaluating Eq. (8) is as follows. The first term depends on $d_{s}$, which can vary depending on whether the particle sits directly on the substrate or whether it sits on the embedded sphere; as described below, we use an average value for this quantity, $\left\langle d_{s}\right\rangle$, to incorporate this effect. For the second term, following previous treatments [27,35,36], we evaluate the sum for the case that the particle is positioned directly above an embedded sphere; the error associated with this approximation will be small because when the particle position deviates significantly from being on top of the embedded sphere, the adhesion force will be dominated by 
(a)

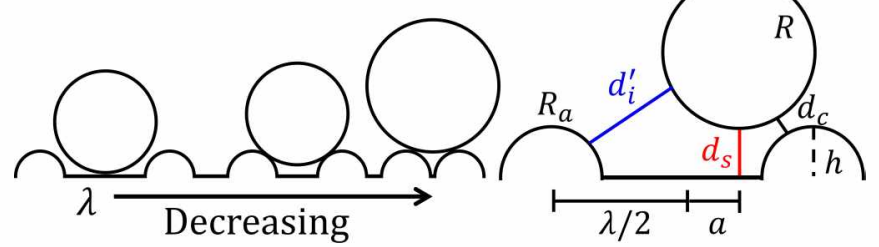

FIG. 2. (a) At large $\lambda$ the particle can sit anywhere on the surface, but at small $\lambda$ the particle must sit atop the embedded spheres. (b) Definition of parameters describing geometry of particle and surface.

the first term (interaction with the substrate) rather than the second term.

As shown in Fig. 2, the particle is separated by contact distance $d_{c}$ from the nearest part of the surface, but its position relative to the substrate, $d_{s}$, can vary with position. When $R$ is large compared to $\lambda$, the particle is always near the top of the embedded spheres such that $d_{s} \approx h+d_{c}$ [23]. In contrast, when $R \lesssim \lambda, d_{s}$ varies with the particle position in the range $d_{c} \leqslant d_{s} \leqslant h+d_{c}$. We find $d_{s}$ as a function of particle position as follows. With the geometry of the system defined according to Fig. 2(b) such that the origin of the $x$ axis is midway between neighboring embedded spheres, the $x$ coordinates of the center of the particle and embedded sphere are $a$ and $\lambda / 2$, respectively. Along the $x$ axis $(y=0)$, the set of positions $d_{c}$ below the bottom surface of the particle is given by

$$
z_{p}=-\sqrt{\left(R+d_{c}\right)^{2}-(x-a)^{2}}+R+d_{c}+d_{s},
$$

while the top surface of the embedded sphere is given by

$$
z_{a}=\sqrt{R_{a}^{2}-(x-\lambda / 2)^{2}}-R_{a}+h
$$

The particle is separated by $d_{c}$ from the embedded sphere at the condition where $z_{a}=z_{p}$ and $d z_{a} / d x=d z_{p} / d x$. Since $d_{s}$ cannot be less than $d_{c}$, if $d_{s}<d_{c}$, the particle directly contacts the substrate rather than an embedded sphere. The solution obtained for $d_{s}$ is

$$
d_{s}=\max \left\{d_{c} ; h-R_{a}-R+\sqrt{\left(R+d_{c}\right)^{2}-\frac{\left(R+d_{c}\right)^{2}\left(a-\frac{\lambda}{2}\right)^{2}}{\left(R+d_{c}-R_{a}\right)^{2}}}+\sqrt{R_{a}^{2}-\frac{R_{a}^{2}\left(a-\frac{\lambda}{2}\right)^{2}}{\left(R+d_{c}-R_{a}\right)^{2}}}\right\} .
$$

Assuming the particle has a uniform probability of adhering at any position $0 \leqslant a \leqslant \lambda / 2$ on the surface, the average $d_{s}$ can be determined,

$$
\left\langle d_{s}\right\rangle=\frac{1}{\lambda / 2} \int_{0}^{\lambda / 2} d_{s} d x .
$$

For a particle positioned directly above an embedded sphere, $d_{i}^{f}$ is given by

$$
d_{i}^{\prime}=\left[\left(R+R_{a}+d_{c}\right)^{2}+x_{a, i}^{2}+y_{a, i}^{2}\right]^{\frac{1}{2}}-R-R_{a} .
$$

While the infinite sum in Eq. (8) converges rapidly, a closed-form analytical model is of more use. We develop such an expression by approximating the terms $i>1$, where $i=1$ is the asperity directly under the particle, in Eq. (8) by an integral,

$$
\sum_{i=1}^{\infty} \frac{z_{i}^{\prime}}{d_{i}^{\prime 3}} \approx \frac{1}{d_{c}^{2}}+\frac{1}{\lambda^{2}} \int_{0}^{2 \pi} \int_{\lambda / 2}^{\infty} \frac{z^{\prime} r}{d^{3}} d r d \theta
$$

where $r$ is the two-dimensional radial coordinate with origin at the center of the particle, and

$$
d^{\prime}=\left[\left(R+R_{a}+d_{c}\right)^{2}+r^{2}\right]^{\frac{1}{2}}-R-R_{a} .
$$

The physical significance of the integral in Eq. (12) is, in essence, to replace discrete pairwise interactions between the particle and asperities, which have a number density $1 / \lambda^{2}$, with a continuum of interactions, where the surface has the same number density of asperities; the integral starts at $\lambda / 2$ to exclude the asperity directly below the particle, which is included explicitly as the first term in the expression.

The contribution to the integral in Eq. (12) is largest for $r \ll R$-because as $r$ becomes comparable to $R$, the underside of the particle has curved significantly away from the surface. In this limit $z^{\prime} \approx d^{\prime}$ and we use a second-order Taylor expansion for $d^{\prime}$ in the limit $r \ll R$, to obtain

$$
d^{\prime} \approx \frac{r^{2}}{2\left(R+R_{a}+d_{c}\right)}+d_{c} .
$$

Using this expression for $d^{\prime}$, the integral in Eq. (12) can be evaluated analytically, and then combined with Eq. (8) to give our final result,

$$
\begin{aligned}
F_{V, \text { model }}= & \frac{H_{\mathrm{Si}} R}{6\left\langle d_{s}^{2}\right\rangle}+\frac{H_{\mathrm{SiO}_{2}} R}{6 d_{c}^{2}}\left\{\frac{1}{1+R / R_{a}}\right. \\
& \left.+\frac{16 \pi d_{c}^{2}\left(R+R_{a}+d_{c}\right)^{2}}{\lambda^{2}\left(1+R / R_{a}\right)\left[\lambda^{2}+8 d_{c}\left(R+R_{a}+d_{c}\right)\right]}\right\} .
\end{aligned}
$$

In Eq. (15), the first term accounts for the noncontact force, i.e., a force where the separation from the particle is greater than $d_{c}$, between the particle and the substrate; the second term accounts for the contact force between the particle and the embedded sphere directly below it; and the third term accounts for the noncontact force between the particle and all other embedded spheres on the surface. Figure 3 depicts the physical significance of the three terms of Eq. (15). We note that physically, the term accounting for the embedded spheres overlaps with the term for the substrate as shown in Fig. 3; however, since these volumes overlap far from the particle, and since the intermolecular van der Waals force decays rapidly with distance, $\left(\sim 1 / r^{7}\right)$, regions of the surface close to the particle dominate the adhesion force causing error from double counting the overlapping regions to be relatively insignificant. 


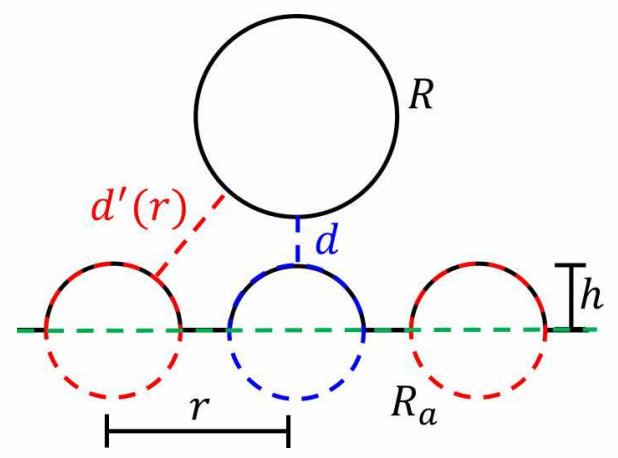

FIG. 3. Our modeled interaction between a spherical particle and the well-defined surface of Ramakrishna et al. [20,39]. In Eq. (15), the second term accounts for contact interaction with the embedded sphere below the particle (blue), and the first and third term account for the noncontact interactions with the substrate (green) and embedded spheres (red), respectively.

\section{B. Comparison of theory with experiments and previous models}

The experiments of Ramakrishna et al. are ideal to validate our analytic expression since the embedded spheres have a well-characterized radius, protrusion height from the substrate, and surface density, and the adhering particle is spherical and smooth. As discussed above, the experiments showed that the adhesion follows JKR contact mechanics at high $\sigma$, but DMT contact mechanics at lower $\sigma$.

We apply our theory by using Eq. (15) with $R=$ $9 \mu \mathrm{m}, R_{a}=6 \mathrm{~nm}$, and $d_{c}=0.3 \mathrm{~nm}$ [20,39]. We calculate Hamaker constants [17] using the dielectric constants $\varepsilon=\{2.3,11.7,1.46,1.8\}$ and indices of refraction $n=$
$\{1.51,3.02,3.9,1.313\}$ for $\left\{\mathrm{PE}, \mathrm{Si}, \mathrm{SiO}_{2}, \mathrm{C}_{10} \mathrm{~F}_{18}\right\}$, and obtain $H_{\mathrm{Si}}=7.552 \times 10^{-20} \mathrm{~J}$ and $H_{\mathrm{SiO}_{2}}=2.357 \times 10^{-20} \mathrm{~J}$ for the PE particle interacting with the $\mathrm{Si}$ substrate and the $\mathrm{SiO}_{2}$ embedded spheres, respectively, within a liquid $\mathrm{C}_{10} \mathrm{~F}_{18}$ medium. We obtain results for the force as a function of the surface density of embedded spheres, $\sigma=1 / \lambda^{2}$.

Figure 4(a) shows the adhesion force on the particle due to the substrate and embedded spheres as calculated by our theory. The force due to interaction with the substrate dominates at very low $\sigma$, where the particle adheres directly to the substrate rather than to the embedded spheres. At all but very low $\sigma$, the particle must adhere near the tops of embedded spheres such that $d_{s}$ is roughly constant at $d_{s} \approx h+d_{c}$, where $h=6 \mathrm{~nm}$. The force due to interactions with the embedded spheres increases as $\sigma$ increases, since at higher $\sigma$ more embedded spheres are in closer proximity to the particle. We note that although the substrate is further from the surface, the force from interaction with the substrate dominates adhesion for most of the $\sigma$ regime because $H_{\mathrm{Si}}$ is $\sim 3$ times larger than $\mathrm{H}_{\mathrm{SiO}_{2}}$.

In Fig. 4(b), we assess the accuracy of our theory with the experimental results; we emphasize that our results do not have any adjustable parameters. Our theory calculates the adhesion force accurately at intermediate $\sigma$, precisely where Ramakrishna et al. [20,39] found that the experimental behavior follows DMT contact mechanics. In contrast, at low and high $\sigma$, where JKR mechanics is expected, our theory is less accurate.

We also assess the accuracy of the widely used Rabinovich and Katainen models, using $R_{a}=6 \mathrm{~nm}, d_{c}=0.3 \mathrm{~nm}$, and $h=6 \mathrm{~nm}$. We adapt their models to include distinct Hamaker constants for the embedded sphere and the substrate force (a)

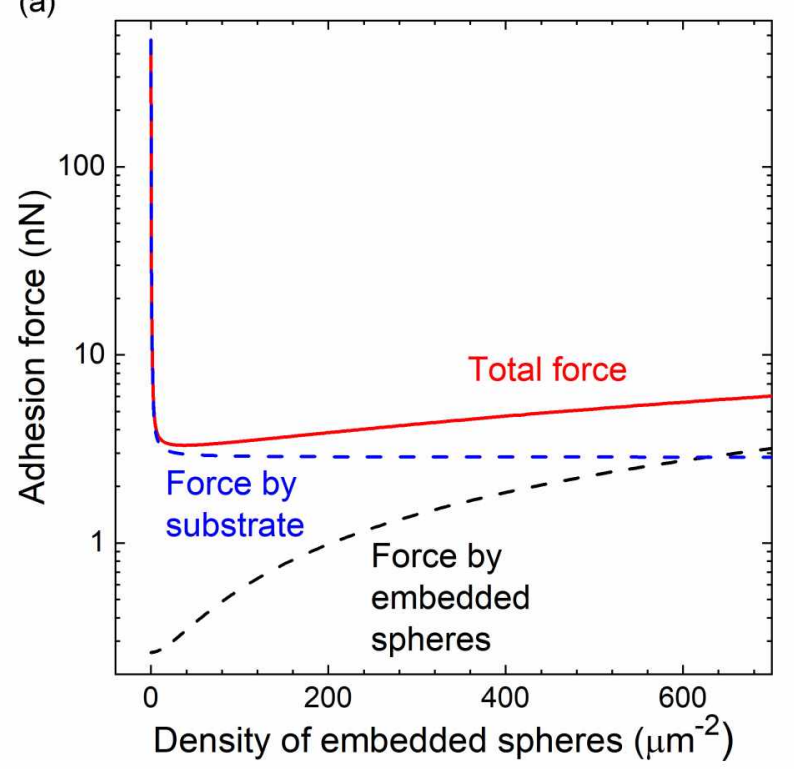

(b)

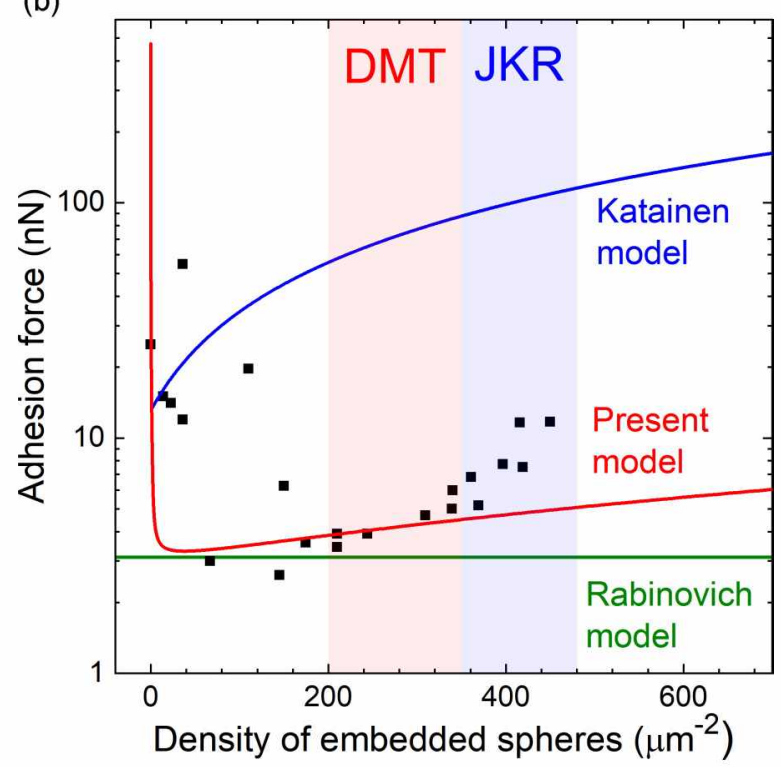

FIG. 4. (a) The total force [red solid line; Eq. (15)], the force from the substrate [blue dashed line; first term of Eq. (15)], and the force from the embedded spheres [black dashed line; last two terms of Eq. (15)] predicted by our proposed theory. Note there are no adjustable parameters in our theory. (b) Experimental results of Ramakrishna et al. [20,39] (black squares) compared with the Rabinovich model [green; Eq. (16)], the Katainen model [blue; Eq. (17)], and our proposed theory [red; Eq. (15)]. The demarcation of the DMT and JKR regimes is approximate, and based on the experimental results of Ramakrishna et al. [39] that showed that the DMT regime applied at $245 \mu \mathrm{m}^{-2}$ while the JKR regime applied at $450 \mu \mathrm{m}^{-2}$. 
contributions,

$$
F_{V, \text { Rabinovich }}=\frac{R}{6 d_{c}^{2}}\left[\frac{H_{\mathrm{SiO}_{2}}}{1+R / R_{a}}+\frac{H_{\mathrm{Si}}}{\left(1+h / d_{c}\right)^{2}}\right],
$$

and

$$
F_{V, \text { Katainen }}=\frac{A_{C}}{6 d_{c}^{2}}\left[H_{\mathrm{SiO}_{2}} \sigma R_{a}+\frac{H_{\mathrm{Si}}}{\pi d_{c}\left(1+h / d_{c}\right)^{3}}\right] .
$$

In the Katainen model, the first term represents the contact forces between the deformed "flat" particle and multiple embedded spheres, and the second term represents the noncontact forces between the deformed particle and the substrate. We estimate the apparent contact area as the particle detaches from the surface, $A_{C}$, for the Katainen model using JKR theory with an applied load of $5 \mathrm{nN}$ as used in the experiments [11],

$$
A_{C}=\pi\left\{\frac{R}{K}\left[P+3 \pi R \gamma+\sqrt{6 \pi R \gamma P+(3 \pi R \gamma)^{2}}\right]\right\}^{\frac{2}{3}},
$$

where $P$ is the applied load; $\gamma$ is the work of adhesion, $0.05 \mathrm{~J} \mathrm{~m}^{-2}$; and $K$ is the effective elastic constant, $0.29 \mathrm{GPa}$ [39].

The present analytic model is seen to be more accurate than these previous analytic models. The Rabinovich model considers only force contributions from the embedded sphere directly below the particle, and so it predicts a constant force with changing $\sigma$. The Katainen model does account for interactions with multiple embedded spheres, and thus predicts that the adhesion force increases with increasing $\sigma$, but it does so in the context of JKR contact mechanics; it therefore will not correctly model the DMT regime, and even in the regime where JKR contact mechanics is relevant, the contact area will be a nontrivial function of the surface roughness (in our application here we used the contact area for a flat surface). Furthermore, both the Rabinovich and Katainen models presume that the particle is positioned above embedded spheres, and therefore does not accurately predict the adhesion force at low $\sigma$ where the particle is more likely positioned between embedded spheres and thus located closer to the substrate.

\section{EXTENSION OF THEORY TO GENERAL ROUGH SURFACES}

Having validated our theory by comparison to the Ramakrishna et al. experimental results, we now adapt our theory to a sinusoidal surface which more closely resembles real rough surfaces. Here, we extend our theory to approximate the adhesion force on a spherical particle due to a sinusoidal surface,

$$
z_{s}(x, y)=A\left[\frac{1}{2} \sin (2 \pi x / \lambda)+\frac{1}{2} \sin (2 \pi y / \lambda)\right],
$$

where $z_{s}$ is the height of the surface as a function of the coordinates $x$ and $y$, and $A$ and $\lambda$ are the amplitude and wavelength of the function describing the surface roughness.

As seen in the experimental results of Ramakrishna et al., when $R \lessgtr \lambda$, the adhesion force strongly depends on the particle position on the rough surface-i.e., the force is very different if the particle sits atop an embedded sphere or directly on the substrate. For the well-characterized surfaces of Ramakrishna et al., we were able to include this case in our

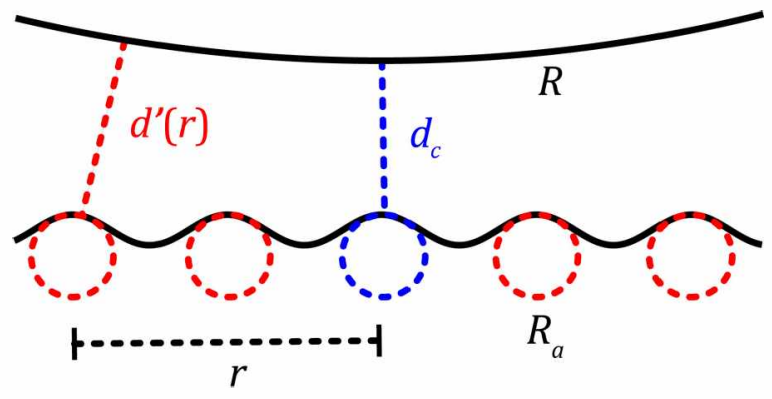

FIG. 5. Our modeled interaction between a sinusoidal surface and sphere. In Eq. (22), the first term accounts for the contact force contribution from the asperity directly under the center of the sphere (in blue) and second term accounts for the noncontact force contributions of all other asperities (in red).

model. However, for real rough surfaces, the effect of particle position when $R \leqslant \lambda$ is even more complex-i.e., the particle could fit snugly into valleys where it experiences enhanced adhesion force, or sit on top of asperities where it experiences reduced adhesion force. Due to the irregular geometry of real rough surfaces, it will be in impossible to rigorously include this effect in a simple model. For this reason, we only address the case when $R \gg \lambda$ such that the particle cannot fit between asperities and must sit above them [23]. We note that satisfaction of this condition is required for correct application of the Rabinovich and Katainen models as well.

To assess the accuracy of our theory, rather than compare our theory to experiments, which have uncertain particle and surface morphologies and deformation on contact, we compare to the exact theoretical van der Waals force calculated in the DMT regime for the well-characterized sinusoidal surface, by explicitly summing interatomic interactions.

\section{A. Theory for general rough surfaces}

Equation (6) describes the adhesion force on a particle by a surface in the continuum approximation. A sinusoidal surface has minima and maxima, with the atoms located near the maxima being closer to the atoms in the particle. Since the van der Waals force between two atoms decays rapidly ( $\sim$ $\left.1 / r^{7}\right)$, the force is dominated by interactions between atoms at the bottom of the particle and atoms near local maxima of the surface. The regions of the surface contributing most to the integral can therefore be approximated by a collection of second-order Taylor expansions around all local maxima, where each maxima, $i$, is described by

$$
z_{s, i}^{\prime}(x, y) \approx A-\frac{4 \pi^{2} A}{\lambda^{2}}\left[\left(x-x_{m, i}\right)^{2}+\left(y-y_{m, i}\right)^{2}\right]
$$

and $x_{m, i}, y_{m, i}$ are the coordinates of local maximum $i$.

Equation (20) also represents the second-order Taylor expansion for a collection of spheres of radius $R_{a}=\lambda^{2} / 2 \pi^{2} A$, with sphere $i$ centered at $\left(x_{m, i}, y_{m, i}, A-R_{a}\right)$ as shown in Fig. 5. Therefore, in the regime where the second-order Taylor expansions are valid, Eq. (6) for a sinusoidal surface is 
equivalent to

$$
F_{V}=\sum_{i=1}^{\infty} \iiint_{X_{p}} \rho_{p} d X_{p} \iiint_{X_{s, i}} \rho_{\mathrm{sp}} \frac{6 C\left(z_{p}-z_{\mathrm{sp}, i}\right)}{r_{i}^{8}} d X_{\mathrm{sp}, i},
$$

where $X_{\mathrm{sp}, i}$ represents the coordinates of a sphere with radius $R_{a}=\lambda^{2} / 2 \pi^{2} A$ centered at $\left(x_{m, i}, y_{m, i}, A-R_{a}\right)$. Equation (21) is the same as the second term in Eq. (6) for the well-defined surface of Ramakrishna et al. Additionally $d_{i}^{\prime}$ is the same as that used in Eq. (11). Thus, following the process detailed in the previous section, Eq. (21) simplifies to the last two terms of Eq. (15),

$$
\begin{aligned}
F_{V, \text { model }}= & F_{V}^{S}\left\{\frac{1}{1+R / R_{a}}\right. \\
& \left.+\frac{16 \pi d_{c}^{2}\left(R+R_{a}+d_{c}\right)^{2}}{\lambda^{2}\left(1+R / R_{a}\right)\left[\lambda^{2}+8 d_{c}\left(R+R_{a}+d_{c}\right)\right]}\right\},
\end{aligned}
$$

where $F_{V}^{S}$ is given by Eq. (2).

Typically, rough surfaces have been characterized by the rms roughness and the wavelength of the roughness. Our theory can be applied to these surfaces by using $\lambda$ as the wavelength and relating $R_{a}$ to the rms roughness. Rabinovich et al. related $R_{a}$ to $\lambda$ and the rms roughness, $\psi$, using a hemispherical close-packed surface such that $R_{a}=\lambda^{2} /(k \psi)$, where $k \approx 58$ [36]. Alternatively, we can relate $\psi$ and $\lambda$ to $R_{a}$ using a sinusoidal surface where $A=2 \psi$ such that $R_{a}=\lambda^{2} /\left(4 \pi^{2} \psi\right)$.

\section{B. Comparison with numerical calculation and previous models}

The van der Waals force, given by Eq. (5), can be calculated numerically by explicitly summing the individual force contributions from all atom pairs in the two objects until converged. We consider the surface and sphere to be composed of crystal arrays of atoms arranged in a cubic lattice with lattice parameter $\delta$; physically $\delta$ is a constant that is approximately $0.3 \mathrm{~nm}$ [17]. In the macroscopic limit, the type of crystal lattice does not affect the functional dependence of the force on the sphere radius, surface roughness, or the particle-surface separation (the type of crystal lattice only affects the magnitude of the Hamaker constant). As is standard procedure in molecular simulations, we exploit the fact that this sum is rapidly converging, and cut off the sum at a finite distance, $r_{\text {cut }}$; we add a constant to the interatomic interactions to make the interatomic force continuous at the cutoff distance,

$$
F_{V}=\sum_{i} \sum_{j} \frac{6 C z_{i j}}{r_{i j}}\left(\frac{1}{r_{i j}^{7}}-\frac{1}{r_{\text {cut }}^{7}}\right) .
$$

To model the sinusoidal surface we include atoms $j$ with $z_{j}<z_{s}\left(x_{j}, y_{j}\right)+0.75 \delta$ where $z_{s}(x, y)$ is given from Eq. (19). We verified our numerical method by comparing scaling behavior of the numerical solution to the analytic solution [Eq. (2)] for a sphere interacting with a flat surface, and finding that our procedure leads to the correct scaling behavior, i.e., $F_{V}^{S} \sim R$ and $F_{V}^{S} \sim d_{c}^{-2}$ at small $d_{c}$.

We assess the accuracy of our theory by comparing with the numerical solution. Figure 6 shows results for $F_{V} / F_{V}^{S}$

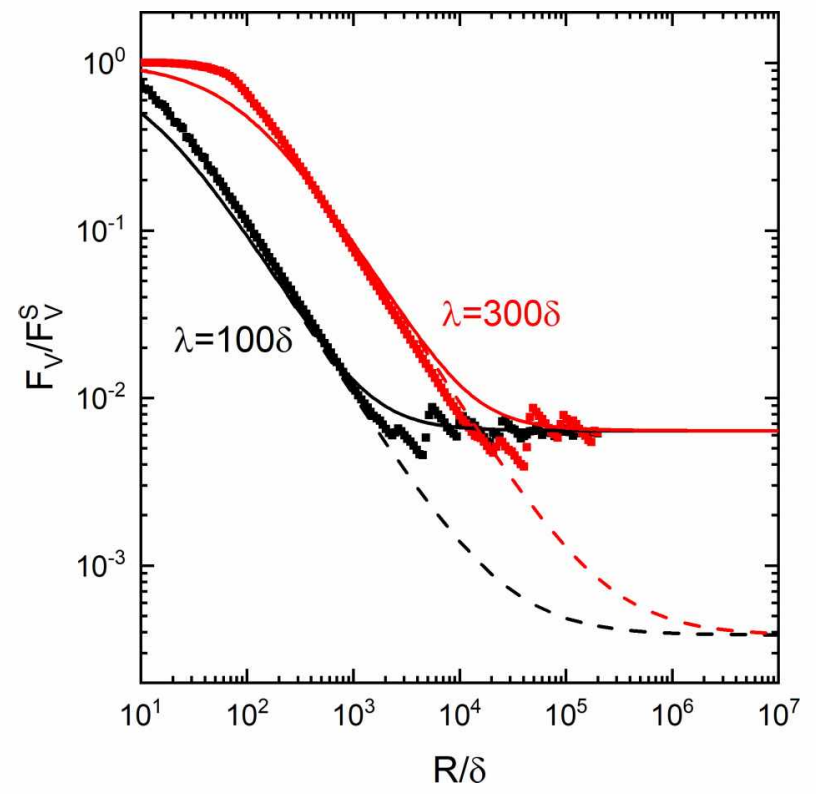

FIG. 6. $F_{V} / F_{V}^{S}$ as a function of $R$ for a particle interacting with a sinusoidal surface with $A=50 \delta$ and $\lambda=100 \delta$ and $300 \delta$, separated by a distance of $1 \delta$. Squares: explicit sum [Eq. (23)] with $r_{\text {cut }}=8 \delta$; Solid line: our proposed theory [Eq. (22)]. Dashed line: Rabinovich model [Eq. (4)].

calculated from the numerical solution [Eq. (23)], our theory [Eq. (22)], and the Rabinovich model [Eq. (4)]. $F_{V}^{S}$ is the force on a particle due to a flat surface, and thus $F_{V} / F_{V}^{S}$ represents the effect of surface roughness on adhesion. In the numerical solution [Eq. (23)], at large $R, F_{V} / F_{V}^{S}$ oscillates with increasing particle size as the particle interacts with additional sets of asperities. We note that discontinuous force changes seen in the numerical solution are due to the discrete representation of the particle in terms of atoms; as $R$ increases, atoms are discontinuously added to the particle, leading to discontinuous increases in force.

We find that our analytic expression matches the numerical solution within a factor of 2 throughout the particle size regime. At small $R$, our model and the Rabinovich model are nearly identical. This is because the first term in our analytic expression, which dominates for small $R$, is the same as the first term in the Rabinovich model describing the interaction between the particle and the asperity directly below it. However, the second term, which dominates for large $R$, is fundamentally different from that of the Rabinovich model since it accounts for particle interactions with multiple asperities.

When $R \gg \lambda$, the Rabinovich model significantly underestimates $F_{V}$. The reason for this underestimation is that the Rabinovich model uses a smooth plane at a surfaceroughness-averaged position to describe the interaction with the surface beyond the one asperity directly below the particle. In actuality, interactions with other asperities become important because the van der Waals force decays quickly $\left(\sim 1 / r^{7}\right)$ with distance. Previous experimental studies have similarly concluded that the Rabinovich model significantly underestimates the van der Waals force when particles interact strongly with other asperities [20,27]. By incorporating the 
interaction between the particle and all asperities on the surface, our model accurately predicts $F_{V}$ at large $R$ in the DMT regime.

\section{CONCLUSION}

We derive an analytic expression for the adhesion force between a spherical particle and a rough surface in the DMT regime, which we validate by comparison to experimental results and exact numerical solutions for well-defined rough surfaces. The key facet that distinguishes our approach is that it incorporates interactions with all asperities on the surface. For this reason, in situations where adhesion is in the DMT regime and the particle size is larger than the length scale of the roughness, our approach predicts adhesion forces considerably better than previous analytic expressions that have been widely used.

\section{ACKNOWLEDGMENTS}

This project was supported by the Institut de Radioprotection et de Sûrete Nucleaire and from the National Science Foundation under Grants No. CBET-1604909 and No. DMR1206480
[1] L. J. Jallo, Y. Chen, J. Bowen, F. Etzler, and R. Dave, J. Adhes. Sci. Technol. 25, 367 (2011).

[2] J. E. Galvin and S. Benyahia, AIChE J. 60, 473 (2014).

[3] E. R. Beach and J. Drelich, J. Adhes. Sci. Technol. 25, 435 (2011).

[4] P. Prokopovich, S. Theodossiades, H. Rahnejat, and D. Hodson, Wear 268, 845 (2010).

[5] S. I. Krasheninnikov, A. Y. Pigarov, R. D. Smirnov, M. Rosenberg, Y. Tanaka, D. J. Benson, T. K. Soboleva, T. D. Rognlien, D. A. Mendis, B. D. Bray, D. L. Rudakov, J. H. Yu, W. P. West, A. L. Roquemore, C. H. Skinner, J. L. Terry, B. Lipschultz, A. Bader, R. S. Granetz, C. S. Pitcher et al., Plasma Phys. Control. Fusion 50, 124054 (2008).

[6] A. Rondeau, S. Peillon, A. Roynette, J. C. Sabroux, T. Gelain, F. Gensdarmes, V. Rohde, C. Grisolia, and E. Chassefière, J. Nuc1. Mater. 463, 873 (2015).

[7] S. Ratynskaia, C. Castaldo, H. Bergsker, and D. Rudakov, Plasma Phys. Control. Fusion 53, 074009 (2011).

[8] K. A. McCarthy, D. A. Petti, W. J. Carmack, and G. R. Smolik, Fusion Eng. Des. 42, 45 (1998).

[9] A. El-Kharbachi, J. Chêne, S. Garcia-Argote, L. Marchetti, F. Martin, F. Miserque, D. Vrel, M. Redolfi, V. Malard, C. Grisolia, and B. Rousseau, Int. J. Hydrogen Energy 39, 10525 (2014).

[10] C. Grisolia, E. Hodille, J. Chene, S. Garcia-Argote, G. Pieters, A. El-Kharbachi, L. Marchetti, F. Martin, F. Miserque, D. Vrel, M. Redolfi, V. Malard, G. Dinescu, T. Acsente, F. Gensdarmes, S. Peillon, B. Pegourié, and B. Rousseau, J. Nucl. Mater. 463, 885 (2015).

[11] K. L. Johnson, K. Kendall, and A. D. Roberts, Proc. R. Soc. Londond, Ser. A 324, 301 (1971).

[12] B. Derjaguin, V. Muller, and Y. Toporov, J. Colloid Interface Sci. 53, 314 (1975).

[13] D. Tabor, J. Colloid Interface Sci. 58, 2 (1977).

[14] K. L. Johnson and J. A. Greenwood, J. Colloid Interface Sci. 192, 326 (1997).

[15] J. S. Marshall and S. Li, Adhesive Particle Flows (Cambridge University Press, New York, 2014).

[16] H. C. Hamaker, Physica (Amsterdam) 4, 1058 (1937).

[17] J. Israelachvili, Intermolecular and Surface Forces, 3rd ed. (Academic Press, New York, 2011).

[18] H. Krupp, Adv. Colloid Interface Sci. 1, 111 (1967).

[19] P. G. C. Petean and M. L. Aguiar, Powder Technol. 274, 67 (2015).
[20] S. N. Ramakrishna, L. Y. Clasohm, A. Rao, and N. D. Spencer, Langmuir 27, 9972 (2011).

[21] T. Kim, C. Min, M. Jung, J. Lee, C. Park, and S. Kang, Appl. Surf. Sci. 389, 889 (2016).

[22] A. Kumar, T. Staedler, and X. Jiang, J. Colloid Interface Sci. 409, 211 (2013).

[23] G. W. Tormoen, J. Drelich, and J. Nalaskowski, J. Adhes. Sci. Technol. 19, 215 (2005).

[24] W. Cheng, P. F. Dunn, and R. M. Brach, J. Adhes. 78, 929 (2002).

[25] B. N. J. Persson and M. Scaraggi, J. Chem. Phys. 141, 124701 (2014).

[26] O. Laitinen, K. Bauer, J. Niinimäki, and U. A. Peuker, Powder Technol. 246, 545 (2013).

[27] J. Katainen, M. Paajanen, E. Ahtola, V. Pore, and J. Lahtinen, J. Colloid Interface Sci. 304, 524 (2006).

[28] E. R. Beach, G. W. Tormoen, J. Drelich, and R. Han, J. Colloid Interface Sci. 247, 84 (2002).

[29] S. Peillon, A. Autricque, M. Redolfi, C. Stancu, F. Gensdarmes, C. Grisolia, and O. Pluchery, J. Aerosol Sci. 137, 105431 (2019).

[30] H. Zhou, M. Götzinger, and W. Peukert, Powder Technol. 135136, 82 (2003).

[31] T. D. B. Jacobs, K. E. Ryan, P. L. Keating, D. S. Grierson, J. A. Lefever, K. T. Turner, J. A. Harrison, and R. W. Carpick, Tribol. Lett. 50, 81 (2013).

[32] Z. Chai, Y. Liu, X. Lu, and D. He, ACS Appl. Mater. Interfaces 6, 3325 (2014).

[33] Q. Li, V. Rudolph, and W. Peukert, Powder Technol. 161, 248 (2006).

[34] S. Matope, Y. I. Rabinovich, and A. F. Van der Merwe, Colloids Surf., A 411, 87 (2012).

[35] H. Rumpf, Particle Technology (Springer, Netherlands, Dordrecht, 1990).

[36] Y. I. Rabinovich, J. J. Adler, A. Ata, R. K. Singh, and B. M. Moudgil, J. Colloid Interface Sci. 232, 10 (2000).

[37] Y. I. Rabinovich, J. J. Adler, A. Ata, R. K. Singh, and B. M. Moudgil, J. Colloid Interface Sci. 232, 17 (2000).

[38] C. Q. LaMarche, S. Leadley, P. Liu, K. M. Kellogg, and C. M. Hrenya, Chem. Eng. Sci. 158, 140 (2017).

[39] S. N. Ramakrishna, P. C. Nalam, L. Y. Clasohm, and N. D. Spencer, Langmuir 29, 175 (2013).

[40] J. A. Greenwood and J. B. P. Williamson, Proc. R. Soc. London, Ser. A 295, 300 (1966). 
[41] P. Prokopovich and S. Perni, Langmuir 26, 17028 (2010).

[42] P. Prokopovich and V. Starov, Adv. Colloid Interface Sci. 168, 210 (2011).

[43] P. Prokopovich and S. Perni, Colloids Surf., A 383, 95 (2011).

[44] B. N. J. Persson, Phys. Rev. Lett. 87, 116101 (2001).

[45] L. Pastewka and M. O. Robbins, Proc. Natl. Acad. Sci. USA 111, 3298 (2014).
[46] B. N. J. Persson, J. Phys.: Condens. Matter 20, 312001 (2008).

[47] N. Mulakaluri and B. N. J. Persson, Ep1 96, 66003 (2011).

[48] T. D. B. Jacobs, T. Junge, and L. Pastewka, Surf. Topogr.: Metrol. Prop. 5, 013001 (2017).

[49] S. Eichenlaub, A. Gelb, and S. Beaudoin, J. Colloid Interface Sci. 280, 289 (2004).

[50] R. P. Jaiswal, G. Kumar, C. M. Kilroy, and S. P. Beaudoin, Langmuir 25, 10612 (2009). 